\title{
FORMAÇÃO E CARACTERIZAÇÃO DE NANOPARTÍCULAS LIPÍDICAS SÓLIDAS REDISPERSÍVEIS CONTENDO COMPOSTOS BIOATIVOS DE Petiveria alliacea L.
}

\author{
P.M. SAMORANO, W.P. OLIVEIRA, C.R.F. SOUZA* \\ Universidade de São Paulo, Faculdade de Ciências Farmacêuticas de Ribeirão Preto, Depto de \\ Ciências Farmacêuticas, Laboratório de P\&D em Processos Farmacêuticos - LAPROFAR \\ *e-mail: souzacrf@gmail.com
}

\section{RESUMO}

A encapsulação é uma tecnologia que vem sendo empregada para solucionar os problemas relacionados com a estabilidade e biodisponibilidade de compostos bioativos através da inclusão do agente ativo em um invólucro protetor, o qual pode ser um filme composto de derivados de carboidratos, gomas, proteínas, lipídios ou polímeros. O objetivo desse trabalho foi o desenvolvimento e a caracterização de micropartículas de base lipídica contendo compostos bioativos de P. alliacea visando o aumento da biodisponibilidade e estabilização de sua atividade biológica. Primeiramente foi realizado um estudo de pré-formulação, para o desenvolvimento das composições lipídicas submetidas a secagem por spray drying. Os parâmetros de secagem foram, temperatura do ar de entrada: $100^{\circ} \mathrm{C}$, vazão do ar de secagem: $60 \mathrm{~m}^{3} / \mathrm{h}$ e vazão de alimentação: $4 \mathrm{~g} / \mathrm{min}$. Os sistemas lipídicos particulados obtidos por spray drying apresentaram teor médio de umidade de 3,59 $\pm 0,63$ e atividade de água de 0,261 $\pm 0,012$. A recuperação do produto apresentou resultados entre $38 \%$ e $58 \%$, e o tamanho médio das partículas antes e após a secagem apresentam valores em escala nanométrica (faixa de 250 e $450 \mathrm{~nm}$ ). Todos os sistemas lipídicos particulados apresentaram solubilidade dos componentes da formulação em torno de 70 a $80 \%$, relativos aos sólidos solúveis em água.

\section{INTRODUÇÃO}

Plantas medicinais e aromáticas são importantes fontes de compostos bioativos com elevado valor terapêutico e nutricional. Entretanto existe uma grande necessidade de desenvolver sistemas que permitam a proteção e liberação dos ativos no alvo farmacológico.

A obtenção de sistemas micro/nano estruturados (nanopartículas poliméricas, nanocápsulas, fitosomas, partículas lipídicas sólidas, etc.) pode conferir inúmeras vantagens a produtos naturais incluindo maior atividade biológica in vivo, aumento da estabilidade, liberação sustentada, redução da degradação devido a ação de fatores físicos e químicos, etc (SARAF, 2010; CORTESROJAS et al., 2014), permitindo o desenvolvimento de produtos inovadores que apresentem potencial para aplicação industrial (setores farmacêutico, químico, alimentício e veterinário). A nanoencapsulação de ativos naturais pode contribuir para o aumento da absorção e do tempo de circulação de compostos fitoquímicos no organismo, possibilitando atingir concentrações plasmáticas ideais para um efeito terapêutico.

Dentre as estratégias que podem ser empregadas na obtenção de sistemas nanoestruturados contento produtos naturais 
incluem-se as nanopartículas lipídicas sólidas e os carreadores lipídicos nanoestruturados.

$\mathrm{Na}$ primeira geração de nanopartículas lipídicas, foram utilizados lipídeos sólidos (à temperatura ambiente), para realizar a encapsulação e, por isso, essas estruturas são chamadas partículas lipídicas sólidas (SLN). Esta técnica consiste na adição do princípio ativo no lipídeo sólido fundido o qual é solidificado com o resfriamento, formando as nanopartículas com o princípio ativo incorporado. Este sistema é especialmente vantajoso para compostos lipofílicos, devido à presença dos lipídios. Entretanto técnicas especiais de preparo, também permitem a incorporação de substâncias hidrossolúveis a estes sistemas (SINGH et al., 2010; BUNJES, 2010; WANG et al., 2010).

Entretanto, o emprego desta tecnologia na encapsulação de fármacos apresenta algumas limitações, como por exemplo: expulsão da droga da SLN; limitação da liberação da droga pela solubilidade desta no lipídio, e a concentração máxima das partículas em dispersão aquosa em torno de 30\% (SINGHAL et al., 2011).

Estas limitações foram contornadas com o desenvolvimento de uma segunda geração de partículas lipídicas, denominadas de carreadores lipídicos nanoestruturados (NLC). Carreadores lipídicos nanoestruturados são formados a partir da combinação de lipídeos sólidos com proporções adequadas de lipídeos líquidos, o que permite incorporar uma maior quantidade de ativo, com menor probabilidade de expulsão do material encapsulado (PARDEIKE et al., 2009).

Assim, o objetivo desse projeto trabalho foi o desenvolvimento e a caracterização de nanopartículas lipídicas sólidas como forma de vetorizar compostos bioativos de $P$. alliacea visando o aumento da disponibilidade e estabilização de sua atividade biológica.

A Petiveria alliacea, é uma planta com comprovada atividade antimicrobiana, antiinflamatória e antitumoral, sendo utilizada na medicina tradicional de vários países. As folhas e raízes da $P$. alliacea contém muitos compostos biologicamente ativos, incluindo flavonoides, triterpenos, esteróides e compostos de enxofre, além de conter um composto de enxofre, o dibenzil trisulfito (DTS), que é associado com várias atividades biológicas, com destaque para a ação antitumoral. Assim, a P. alliacea é uma ótima candidata para o desenvolvimento de sistemas micro e/ou nanoestruturados que permitam a veiculação dos compostos bioativos, aumento da estabilidade e da atividade biológica.

Três métodos de secagem são normalmente utilizados para conferir maior estabilidade as nanopartículas lipídicas, são eles: freeze drying, secagem por leito fluidizado e spray drying; sendo este último escolhido para o desenvolvimento deste trabalho (CORTES-ROJAS et al., 2014; INGVARSSON et al., 2011).

\section{MATERIAL E MÉTODOS}

\subsection{Matéria-prima vegetal}

Partes aéreas de Petiveria alliacea, coletadas na cidade de Ubarana, interior de São Paulo (21ํ9'34.6"S 4943'06.3"W).

\subsection{Lipídeos e agentes emulsificante}

Os lipídeos selecionados para a produção das NLC foram gentilmente fornecidos pela empresa Gattefossé, França. Lipídios sólidos: compritol 888 CG ATO (behenato de glicerilo), precirol ATO 5 (gliceril diestearato) e, gelucire 50/13 pellets (estearoil-macrogol-32-gliceridos).

Lipídios líquidos: labrasol (PEG-8 caprilico/caprico glicerideos) e plurol oleique CC 497 (poligliceril-3-dioleato).

\subsection{Tensoativos}

Os tensoativos utilizados para a estabilização das formulações foram: 
- Polisorbato 80 (Tween 80): tensoativo não iônico com EHL de 15 utilizado para obtenção de emulsões o/a (óleo/água).

- Monooleato de sorbitano (Span 80): tensoativo não iônico com valor EHL de 4,3 utilizando como emulsificante lipofílico formando emulsões a/o (água/óleo).

\subsection{Carreadores de secagem}

Foram avaliados como carreadores de secagem os seguintes materiais:

- Fibregum B (goma arábica), Nexira Brasil.

- Aerosil 200 (dióxido de silício coloidal), Evonik-Degussa.

- Lacprodan 80 (concentrado proteico do soro do leite), Arla Foods Ingredients.

\subsection{Métodos experimentais}

\subsubsection{Solução extrativa e extrato concentrado}

A solução extrativa foi preparada de acordo com Guedes (2010), por maceração dinâmica, tendo como solvente extrator uma solução de etanol $70 \%$ (v/v), proporção de $1: 10(\mathrm{p} / \mathrm{v})$ planta/solvente e temperatura de extração de $50{ }^{\circ} \mathrm{C}$, por 90 minutos. Após a obtenção da solução extrativa esta foi concentrada em evaporador rotativo, a temperatura de $50{ }^{\circ} \mathrm{C}$ e pressão de 600 $\mathrm{mmHg}$. $\mathrm{O}$ extrato concentrado foi caracterizado através da determinação do teor de sólidos, densidade, $\mathrm{pH}$ e concentração de polifenóis e polissulfetos.

2.5.2 Desenvolvimento da formulação contendo bioativos de $P$. alliacea

Realizou-se um screening de formulações preparando-se nanopartículas lipídicas sólidas (SLN) e carreadores lipídicos nanoestruturados (NLC), sendo que as SLN apresentam apenas o lipídio sólido em sua composição e as NLC são formuladas através de uma razão entre lipídio sólido e líquido de forma a melhorar a estruturação do sistema e evitar extravasamento de bioativos. As proporções dos tensoativos utilizado para estabilizar as formulação foram determinadas através no EHL (equilíbrio hidrófilo-lipófilo) de cada emulsão.

Com base nos resultados de estabilidade das emulsões obtidas, selecionou-se a composição mais estável para realização dos estudos de secagem por spray drying. Para os estudos do processo de secagem, foi elaborado um planejamento visando avaliar a influencia do tipo do carreador de secagem (isolado e mistura) e a proporção de carreador adicionado a formulação (Tabela 1).

Tabela 1 - Formulações analisadas e submetidas ao processo de secagem por spray drying.

\begin{tabular}{ccccccc}
\cline { 2 - 6 } & F1 & F2 & F3 & FA & FB & FC \\
\hline Gelucire ( \% ) & 7 & 7 & 7 & 7 & 7 & 7 \\
Plurol ( \% ) & 3 & 3 & 3 & 3 & 3 & 3 \\
Extrato conc* ( \% ) & 2 & 2 & 2 & 2 & 2 & 2 \\
Span 80 ( \% ) & 3,26 & 3,26 & 3,26 & 3,26 & 3,26 & 3,26 \\
Tween 80 ( \% ) & 0,74 & 0,74 & 0,74 & 0,74 & 0,74 & 0,74 \\
GA ( \% ) & 8 & - & 4 & 16 & - & 8 \\
WPC ( \% ) & - & 8 & 4 & - & 16 & 8 \\
Aerosil ( \% ) & 2 & 2 & 2 & 2 & 2 & 2 \\
Água ( mL ) q.S.p & 100 & 100 & 100 & 100 & 100 & 100 \\
Amplitude ultrasom ( \% ) & 70 & 70 & 70 & 70 & 70 & 70 \\
\hline
\end{tabular}

* calculado com base no teor de sólidos do extrato concentrado; GA: goma arábica; WPC: proteína concentrada do soro do leite; q.s.p: quantidade suficiente para 
O preparo das formulações foi realizado em três fases distintas. A fase aquosa (FAq) foi composta de água, extrato concentrado de $P$. alliacea e tensoativo hidrofílico (tween 80), a fase oleosa (FO) foi composta por lipídio sólido e líquido e tensoativo lipofílico (span 80), e uma terceira fase, composta pelo carreador de secagem hidratado previamente (goma arábica, concentrado proteico do soro do leite ou a mistura de ambos na proporção de 1:1). Todos os componente foram aquecidos em placa de aquecimento a temperatura de $10{ }^{\circ} \mathrm{C}$ acima do ponto de fusão dos lipídicos sólidos utilizados, sob constante agitação. Quando esta temperatura foi atingida, a FAq foi vertida sobre a FO, formando a pré-emulsão e posteriormente foi adicionado a fase contendo os carreadores de secagem. A pré-emulsão foi então agitada em homogeneizador de alta velocidade (processador ultra-turrax T18 IKA) na velocidade de $1800 \mathrm{rpm}$ por 5 minutos e submetida a processador ultrassônico VCX750 (SONICS Vibracell, Newtown, EUA), com auxílio de uma sonda Tapered microtip 1/4" (cód. 630-0420) com uma frequência de $20 \mathrm{kHz}$, e amplitude de $70 \%$ para obtenção das nanopartículas. Todas as amostras foram resfriadas a temperatura ambiente sob agitação magnética antes de serem submetidas ao processo de secagem.

\subsubsection{Obtenção dos sistemas lipídicos} nanoestruturados secos por spray drying

Para os estudos de secagem, selecionouse a formulação composta por gelucire 50/13 (lipídio sólido), plurol oleique (lipídio líquido), extrato concentrado de P. alliacea (o extrato liofilizado apresentava características higroscópicas e de difícil incorporação nos componentes da formulação, problema que foi solucionado pela adição do extrato concentrado sendo o teor calculado em base de sólidos totais), mistura de tensoativos hidrofílico e lipofílico (tween 80 e span 80), sendo avaliada a influência do tipo e proporção do carreador de secagem adicionado (Tabela 1). Foram investigados a adição de goma arábica, concentrado proteico do soro do leite, mistura dos dois excipiente (1:1), e a concentração de adjuvante adicionado a formulação (8 \% e $16 \%)$.

Após a obtenção e caracterização das composições lipídicas contendo bioativos de Petiveria alliacea (Tabela 1), procedeu-se a secagem por spray drying, avaliando-se as características dos produtos nanoparticulados obtidos.

A secagem foi realizada em um spray dryer de bancada modelo SD 05, fabricado pela LAB-PLANT, Reino Unido. A câmara de secagem possui diâmetro de $215 \mathrm{~mm}$ e altura de $500 \mathrm{~mm}$. Os principais componentes do sistema são: sistema de alimentação de suspensão, composto por uma bomba peristáltica, e um atomizador de duplo fluido a ar comprimido (diâmetro do bico atomizador de $0,5 \mathrm{~mm})$; sistema de alimentação do ar de secagem (compressor e filtro de ar); sistema de controle de temperatura do gás de secagem e sistema coletor do produto seco (ciclone).

A seleção das condições operacionais de secagem foi baseada em estudos anteriores desenvolvidos no LAPROFAR (FERNANDES, 2013; CORTÉS-ROJAS, OLIVEIRA， 2012; GUEDES; SOUZA; OLIVEIRA, 2009; SOUZA, 2003). Os parâmetros operacionais do secador foram fixados em: temperatura de entrada do ar de secagem $\left(\mathrm{T}_{\mathrm{ge}}\right)$ : $90{ }^{\circ} \mathrm{C}$; vazão do ar de secagem $\left(\mathrm{W}_{\mathrm{g}}\right): 60 \mathrm{~m}^{3} / \mathrm{h}$; vazão de alimentação da amostra $\left(\mathrm{W}_{\text {susp }}\right): 4 \mathrm{~g} / \mathrm{min}$; pressão do ar de atomização $\left(\mathrm{P}_{\mathrm{atm}}\right): 3 \mathrm{kgf} / \mathrm{cm}^{2}$, e vazão do ar de atomização $\left(\mathrm{W}_{\mathrm{atm}}\right): 17 \mathrm{lpm}$.

Durante o experimento, variáveis como temperatura e umidade do ar ambiente, temperatura do ar de saída foram monitoradas de forma a se avaliar o desempenho do secador.

A recuperação do produto ou $\mathrm{o}$ rendimento do processo (RP) foi estimada 
por balanço de massas através da relação percentual entre a massa de pó total coletada e a massa de sólidos alimentada no secador (SOUZA; OLIVEIRA, 2012; CORTESROJAS; OLIVEIRA, 2012).

2.5.4 Caracterização física e química dos sistemas nanoparticulados

Os sistemas nanoparticulados obtidos foram caracterizados quanto ao teor de umidade (Karl Fischer), atividade de água, densidade aparente, de compactação e índices de fluidez (Índice de Carr e Fator de Hausner), solubilidade das partículas em água, tamanho de partículas e análise microscópica. As metodologias empregadas nestas determinações estão descritas a seguir.

- Teor de umidade: foi determinado através da titulação pelo método de Karl Fischer (Methrom 870 KF Titrino Plus) de acordo com Fernandes (2013).

- Atividade de água: determinada em triplicata no equipamento AquaLab 4 TEV (Decagon, Devices) à temperatura de $25^{\circ} \mathrm{C}$, utilizando o sensor de ponto de orvalho.

- Densidade aparente e de compactação: amostras dos sistemas lipídicos particulados foram colocados em uma proveta de $10 \mathrm{~mL}$. A massa inicial deste volume foi medida, e empregada na determinação da densidade aparente. Para se determinar a densidade de compactação, a amostra contida na proveta de $10 \mathrm{~mL}$ foi submetida a 1250 quedas em superfície lisa e altura de 3,0 $\pm 0,2 \mathrm{~mm}$ com o auxílio do equipamento Calleva ${ }^{\circledR}$ TDT22 (Frankfurt, Germany) (FERNANDES, 2013).

- Índices de compactação dos pós: foram determinados através da razão de Hausner (RH) e índice de Carr (IC). O FH foi determinado pelo quociente entre a densidade de compactação e a densidade aparente da formulação seca (Equação 1). O IC foi expresso pelo quociente da diferença das densidades aparente e de compactação pela densidade aparente. O IC foi expresso em porcentagem, multiplicando-se o valor obtido por 100, conforme apresentado na Equação 2 (CORTÉS-ROJAS et al., 2014).

$$
\begin{aligned}
& \mathrm{RH}=\frac{\rho \mathrm{c}}{\rho \mathrm{a}} \\
& \mathrm{IC}=\frac{(\rho \mathrm{c}-\rho \mathrm{a})}{\rho \mathrm{c}} \times 100
\end{aligned}
$$

Onde: $\rho$ c é a densidade de compactação $\left(\mathrm{g} / \mathrm{cm}^{3}\right)$ e $\rho$ é a densidade aparente $\left(\mathrm{g} / \mathrm{cm}^{3}\right)$.

- Solubilidade das partículas em água: foi determinada pelo método de Eastman e Moore (1984), com modificações. Amostras de $0,5 \mathrm{~g}$ foram dispersas em $50 \mathrm{~mL}$ de água em agitação magnética por $10 \mathrm{~min} e$ centrifugada a $2330 \mathrm{rcf}$ por $5 \mathrm{~min}$. Alíquotas de $15 \mathrm{~mL}$ do sobrenadante foram secas em estufa a $105^{\circ} \mathrm{C}$ até peso constante. A solubilidade das nanopartículas em água foi calculada com base na massa inicial da amostra solubilizada na alíquota de $15 \mathrm{~mL}$ do sobrenadante. O resultado foi expresso em porcentagem e calculado pela Equação 3.

$\%$ Solubilida de $=\frac{\mathrm{m}_{\text {residual }}}{\mathrm{V}_{\mathrm{al}}} \times \mathrm{V}_{0} \times \frac{1}{\mathrm{~m}_{\text {inicial }}} \times 100$

Onde: $\mathrm{m}_{\text {residual }}$ :massa do resíduo seco, $\mathrm{v}_{\mathrm{al}}$ : volume da alíquota, $\mathrm{v}_{\mathrm{o}}$ : volume inicial, $\mathrm{m}_{\text {inicial }}$ : massa inicial.

- Tamanho de partículas e índice de polidispersão: a determinação do tamanho de partículas e do índice de polidispersão dos sistemas lipídicos nanoestruturados secos foi realizada por espectroscopia de correlação de fótons com o auxílio do equipamento Zetasizer SN Nano 90 (Malvern Instruments, UK). Amostra de $25 \mathrm{mg}$ dos sistemas lipídicos secos foi dispersa em $15 \mathrm{~mL}$ de água com o auxílio do processador ultrassônico.

As formulações secas também foram analisadas por microscopia óptica e análise de imagens (Image Pro-Plus). Amostras secas foram dispersas em água:propilenoglicol (1:1) 
colocadas em lâmina de vidro e analisadas em microscópio Olympus $^{\circledR}$ - modelo BX60MIV.

\section{RESULTADOS E DISCUSSÃO}

\subsection{Caracterização do extrato concentrado}

$\mathrm{O}$ extrato concentrado foi caracterizado de acordo com seu teor de sólidos, densidade, $\mathrm{pH}$, teor alcoólico, polifenóis totais (expressos por ácido gálico) e polissulfetos totais (expresso por DDS - dibenzil dissulfeto). A Tabela 2 apresenta os resultados obtidos.

Tabela 2 - Resultados da caracterização físicoquímica do extrato concentrado (EC).

\begin{tabular}{cc}
\hline Caracterização realizada & EC \\
\hline Teor de sólidos $(\%)$ & $7,47 \pm 0,31$ \\
Densidade $\left(\mathrm{g} / \mathrm{cm}^{3}\right)$ & $1,01 \pm 0,00$ \\
$\mathrm{pH}(-)$ & $6,73 \pm 0,01$ \\
Teor alcoólico ( $\left.{ }^{\circ} \mathrm{GL}\right)$ & 0 \\
$\quad$ Polifenóis totais & $2,01 \pm 0,12$ \\
(mg ac. gálico/g de planta) & \\
Polissulfetos totais & $13,00 \pm 0,32$ \\
(mg DDS/g de planta)
\end{tabular}

\subsection{Obtenção dos sistemas lipídicos nanoestruturados secos por spray drying}

3.2.1 Influencia do tipo e proporção de carreador de secagem

Todas as formulações apresentam a mesma composição lipídica, mesmo teor de extrato concentrado, tensoativos, sílica e água (Tabela 1). A diferença entre elas é que as formulações F1 e F2 apresentam 8\% de carreador de secagem, goma arábica (GA) no caso da $\mathrm{F} 1$ e concentrado proteico do soro do leite (WPC) no caso da F2, enquanto que, as formulações FA e FB apresentam 16\% de GA e $16 \%$ de WPC respectivamente. As formulações F3 (8\%) e FC (16\%), apresentam um blend na proporção de 1:1 de GA e WPC na sua composição. A Figura 2 apresenta o aspecto visual dos produtos da secagem.

Figura 2 - Aspecto dos sistemas nanoparticulados secos por spray drying contendo WPC e/ou GA.

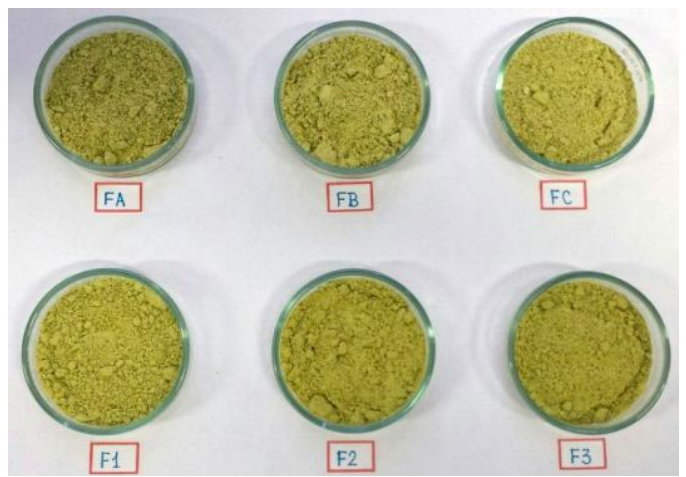

Os sistemas lipídicos nanoestruturados obtidos por spray drying foram analisados quanto ao teor de umidade (Karl Fischer), atividade de água, distribuição granulométrica e tamanho de partículas, densidade aparente e de compactação e índices de fluidez e teste de solubilidade das partículas em água (redispersão). Avaliou-se o desempenho do processo de secagem por medidas do rendimento de produto e monitoramento das temperaturas de saída do ar de secagem e de partes internas do secador (câmara de secagem e ciclone). Os resultados obtidos serão apresentados e discutidos a seguir.

- teor de umidade e atividade de água

A Tabela 3 apresenta os resultados de teor de umidade e atividade de água dos sistemas particulados obtidos.

Tabela 3 - Teor de umidade e atividade de água.

\begin{tabular}{ccc}
\cline { 2 - 3 } & $\begin{array}{c}\text { Teor de umidade } \\
(\boldsymbol{\%})\end{array}$ & $\begin{array}{c}\text { Atividade de água } \\
(-)\end{array}$ \\
\hline F1 & $3,38 \pm 0,04$ & $0,279 \pm 0,03$ \\
F2 & $2,87 \pm 0,11$ & $0,249 \pm 0,01$ \\
F3 & $3,21 \pm 0,06$ & $0,266 \pm 0,01$ \\
FA & $4,67 \pm 0,16$ & $0,263 \pm 0,02$ \\
FB & $3,50 \pm 0,15$ & $0,261 \pm 0,01$ \\
FC & $3,88 \pm 0,18$ & $0,246 \pm 0,01$ \\
\hline
\end{tabular}


A atividade de água do produto indica o teor de água livre na amostra, ou seja, a água que está disponível para reações de degradação do produto. Este parâmetro pode variar de 0 a 1 sendo que, quanto maior for este valor, maior será a quantidade de água disponível para reações físicas, químicas e crescimento microbiano (FERNANDES, 2013). Valores de atividade de água inferiores a 0,5 impede o crescimento microbiano, e abaixo de 0,2 aproximadamente ocorre o favorecimento de reações de oxidação de lipídios. Assim, os resultados apresentados na Tabela 3, evidenciam que as amostras apresentam valores de atividade de água adequados para a manutenção da estabilidade física e microbiológica do produto.

Com relação ao teor de umidade, é preconizado um valor máximo de $10 \%$ para formas farmacêuticas sólidas. Assim, os produto gerados apresentam valores ideais de umidade favorecendo a estabilidade do produto.

\section{- Tamanho de partículas e índice de polidispersão}

A Figura 3 mostra que antes e após a secagem o tamanho das partículas encontramse na faixa nanométrica (faixa de 250 e 450 $\mathrm{nm})$. Nas amostras líquidas as partículas obtidas foram menores que nas amostras redispersas. Observou-se que em menores proporções de carreadores as partículas obtidas apresentaram menores tamanhos, independente do carreador.

A Figura 4 representa o índice de polidispersão das amostras líquidas e secas. Observando os resultados plotados é possível concluir que apenas a formulação FB líquida apresentou o índice recomendado de pdI para sistemas nanopartículados farmacêuticos (menor que 0,3).
Figura 3 - Tamanho das partículas antes (dispersões líquidas) e após o processo de secagem (amostras redispersas).

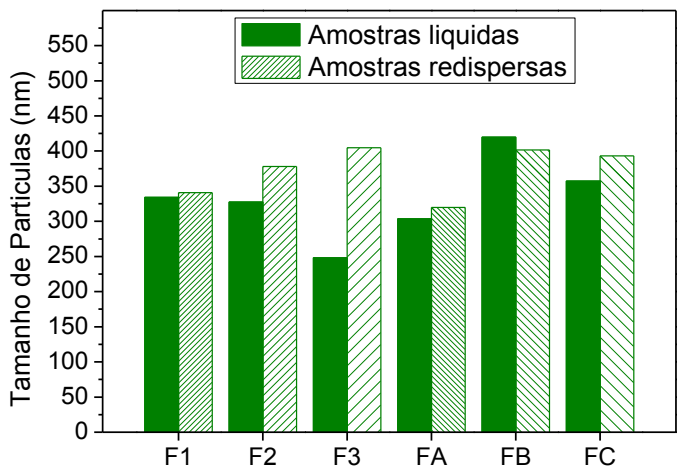

Figura 4 - Índice de polidispersão antes (dispersões líquidas) e após (amostras redispersas) o processo de secagem por spray drying.

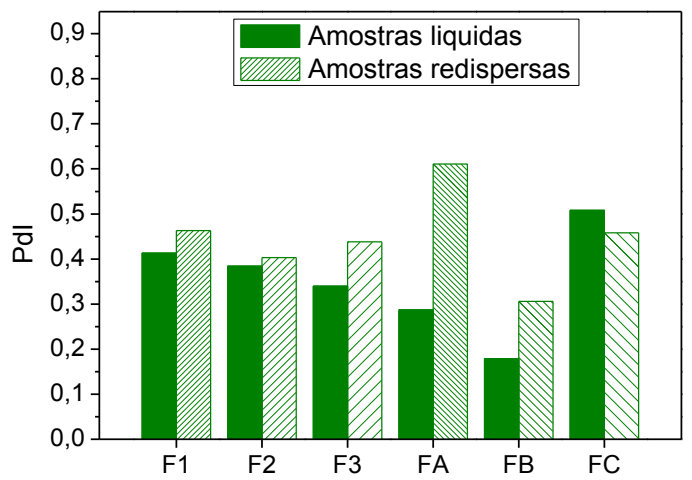

\section{- Distribuição granulomética e tamanho médio de partículas}

O tamanho de partícula é um importante parâmetro pois está diretamente relacionado à solubilidade e fluidez do produto, propriedades importantes para aplicações industriais. A partir de imagens obtidas em microscópio óptico e utilizando-se o software analisador de imagens (Image Pro-Plus ${ }^{\circledR}$ ) foi possível determinar o diâmetro médio e a distribuição granulométrica dos sistemas particulados obtidos. Os gráficos da distribuição granulométrica (dados não apresentados) mostram que os sistemas lipídicos microparticulados são compostos de partículas polidispersas e apresentam diâmetro médio de partículas em torno de 3,5 
micras. A Tabela 4 apresenta os valores de tamanho médio de partículas (desvio padrão).

\section{- Densidade e índices de fluidez}

Os sistemas lipídicos secos obtidos também foram avaliadas quanto as suas propriedades farmacotécnicas através da determinação da densidade aparente (ou bulk), densidade de compactação, razão de Hausner (RH) e índice de Carr (IC). A RH, assim como o IC, são parâmetros que permitem avaliar as características de fluidez e de compressibilidade de pós (Tabela 4).
Analisando a Tabela 4 é possível concluir que apenas a F2 apresenta boa capacidade de compressão $(\mathrm{FH}<1,18)$. Em relação ao índice de Carr, materiais com índice entre 5 e $15 \%$ são considerados de fluidez excelente, de 12 a $16 \%$ fluxo bom, de 18 a $21 \%$ escasso, de 22 a $35 \%$ fluxo fraco, de 35 a $38 \%$ muito fraco e maior que $40 \%$ extremamente fraco (AULTON, 2005). Por esta razão, as dispersões lipídicas F3, FB e FC são caracterizadas como de fluidez escassa, a F1 e a FA são consideradas de fluxo fraco, e a F2 é considerada de excelente fluidez.

Tabela 4 - Tamanho médio de partículas e características farmacotécnicas dos sistemas microparticulados.

\begin{tabular}{cccccc}
\hline Formulações & $\begin{array}{c}\mathbf{D p} \\
(\boldsymbol{\mu m})\end{array}$ & $\begin{array}{c}\mathbf{d}_{\mathbf{a}} \\
\left(\mathbf{g} / \mathbf{c m}^{\mathbf{3}}\right)\end{array}$ & $\begin{array}{c}\mathbf{d}_{\mathbf{c}} \\
\left(\mathbf{g} / \mathbf{c m}^{\mathbf{3}}\right)\end{array}$ & $\begin{array}{c}\mathbf{F H} \\
(-)\end{array}$ & $\begin{array}{c}\text { IC } \\
(-)\end{array}$ \\
\hline F1 & $3,31 \pm 0,31$ & 0,230 & 0,284 & 1,23 & 23,33 \\
F2 & $3,54 \pm 0,55$ & 0,228 & 0,257 & 1,13 & 12,50 \\
F3 & $4,01 \pm 0,11$ & 0,208 & 0,249 & 1,20 & 20,00 \\
FA & $2,51 \pm 0,39$ & 0,231 & 0,306 & 1,32 & 32,14 \\
FB & $3,24 \pm 0,26$ & 0,204 & 0,246 & 1,21 & 20,69 \\
FC & $3,73 \pm 0,20$ & 0,215 & 0,260 & 1,21 & 20,69 \\
\hline
\end{tabular}

Dp: tamanho médio de partícula; $\mathrm{d}_{\mathrm{a}}$ : densidade aparente; $\mathrm{d}_{\mathrm{c}}$ : densidade compactação; FH: razão de Hausner; IC: índice de Carr

\section{- Solubilidade das formulações secas em água (teste de reconstituição)}

As formulações secas por spray drying foram submetidas a ensaio de reconstituição em água, de forma a avaliar a capacidade destas de serem redispersa novamente assumindo as características da formulação original. Os resultados obtidos estão apresentados na Figura 5.

Analisando os resultados mostrados na Figura 5 pode-se notar que todas as amostras apresentaram solubilidade dos componentes da formulação em torno de 70 a $80 \%$, valores relativos aos sólidos solúveis em água. Estima-se que os particulados não solúveis (precipitados pela centrifugação) são referentes aos adjuvantes de secagem adicionados as formulações, em especial ao dióxido de silício coloidal que é insolúvel em água. Entretanto, o aspecto visual das formulações resuspensas ficou muito similar ao original (formulações antes do processo de secagem), o que comprova que os sistemas lipídicos nanoestruturados obtidos podem ser desidratados e resuspendidos no momento do uso, servindo como uma matéria prima única (contendo o bioativo) para preparação de emulsões com a finalidade desejada.

\section{- Desempenho da secagem por spray drying \\ Para o estudo do processo de secagem, os parâmetros operacionais foram fixados, sendo estudada a influência da composição das formulações de secagem. A Tabela 5 apresenta os resultados experimentais obtidos.}


Figura 5 - Solubilidade (redispersão) em água das formulações secas.

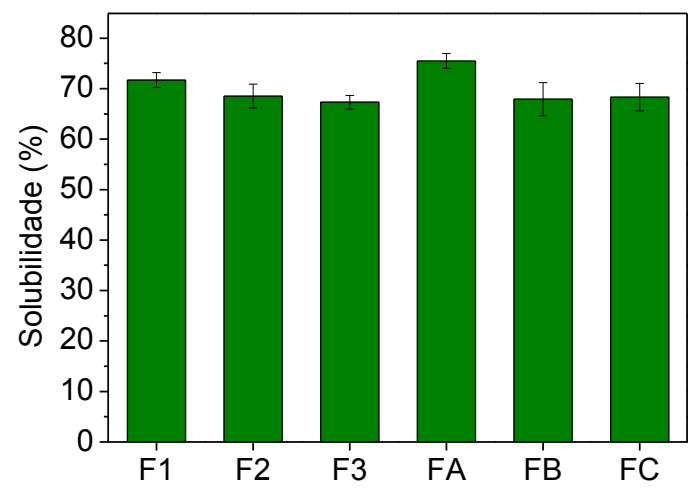

Tabela 5 - Resultados do processo de secagem.

\begin{tabular}{ccccc}
\hline Formulação & $\begin{array}{c}\mathbf{W s}_{\text {real }} \\
(\mathbf{g} / \mathbf{m i n})\end{array}$ & $\begin{array}{c}\mathbf{T}_{\mathbf{g s}} \\
\left({ }^{\circ} \mathbf{C}\right)\end{array}$ & $\begin{array}{c}\text { UR } \\
(\boldsymbol{\%})\end{array}$ & $\begin{array}{c}\mathbf{R P} \\
(\boldsymbol{\%})\end{array}$ \\
\hline F1 & 4,3 & 66,2 & 57,2 & 58,7 \\
F2 & 4,6 & 65,5 & 53,1 & 46,1 \\
F3 & 4,4 & 65,5 & 52,1 & 44,6 \\
FA & 4,1 & 67,9 & 52,8 & 38,0 \\
FB & 4,0 & 66,7 & 64,6 & 48,3 \\
FC & 4,6 & 65,3 & 54,0 & 40,0 \\
\hline
\end{tabular}

$\mathrm{WS}_{\text {real }}$ : vazão de alimentação real, $\mathrm{T}_{\mathrm{gs}}$ : temperatura do ar de saída, UR: umidade relativa, RP: recuperação do produto.

A Tabela 5 mostra que a temperatura média do ar de saída se manteve praticamente constante em todos as secagem realizadas em torno de 65 a $68{ }^{\circ} \mathrm{C}$. Observa-se uma tendência de maior recuperação de produto para as formulações com menores proporções de carreadores, com exceção da formulação FB composta por maior proporção de carreador (WPC).

\section{CONCLUSÕES}

Os sistemas lipídicos nanoparticulados obtidos por spray drying apresentaram valores de teor de umidade médio de 3,59 $\pm 0,63$ e atividade de água de 0,261 $\pm 0,012$, valores adequados para produtos farmacêuticos.
A recuperação do produto apresentou resultados entre $38 \%$ e $58 \%$, considerados adequados para a secagem de formulações lipídicas. Antes e após a secagem o tamanho de partículas se encontravam na escala nanométrica (faixa de 250 e $450 \mathrm{~nm}$ ). O tamanho médio das partículas nas amostras líquidas foram um pouco inferiores às amostras redispersas. Em geral, a redução na concentração dos carreadores de secagem, gerou partículas de menor tamanho médio.

Os gráficos da distribuição granulométrica (dados não apresentados) mostram que os sistemas lipídicos microparticulados são compostos de partículas polidispersas, com diâmetro médio de partículas em torno de 3,5 micras.

Os sistemas lipídico particulados F3, FB e FC foram caracterizadas como de fluidez escassa, o F1 e FA de fluxo fraco, enquanto que apenas a composição F2 foi classificada como de fluidez excelente.

Todos os sistemas lipídicos desidratados apresentaram solubilidade dos componentes da formulação em torno de 70 a $80 \%$, relativos aos sólidos solúveis em água.

\section{REFERÊNCIAS}

AULTON, M. E. Delineamento de formas farmacêuticas. 2 ed. Artmed, 2005.

BUNJES, H. Lipid nanoparticles for the delivery of poorly water-soluble drugs. J. Pharm. Pharmacol., v. 62, p. 1637-1645, 2010 .

CORTÉS-ROJAS, D.F.; OLIVEIRA, W.P. Physicochemical properties of phytopharmaceutical preparations as affected by drying methods and carriers. Drying Technology, v. 30, n. 9, p. 921-934, 2012.

CORTÉS-ROJAS, D.F.; SOUZA, C.R.F.; OLIVEIRA, W.P. Encapsulation of eugenol rich clove extract in solid lipid carriers. J. Food Engineering, v. 127, p.34-42, 2014. 
EASTMAN, J.E.; MOORE, C.O. Cold water soluble granular starch for gelled food composition. US Patent, 4, 465-702, 1984.

FERNANDES, M.R.V. Obtenção, padronização e avaliação biológica de extratos secos a partir da espécie Psidium guajava L. pelo processo spray drying. Tese. FCFRP - USP, 2013, 237p.

GUEDES, R.C. Extratos secos nebulizados de Petiveria alliacea (Phytolaccaceae): Desenvolvimento, padronização e avaliação da atividade antimicrobiana. Relatório Final de IC FAPESP, 2010, 69p.

GUEDES， R.C.M.; NOGUEIRA， N.G.P.; FUSCO-ALMEIDA, A.M.; SOUZA, C.R.F.; OLIVEIRA, W.P. Atividade antimicrobiana de extratos brutos de Petiveria alliacea L. Latim American J. Pharmacy, v. 28 (4), p. 520-524, 2009.

INGVARSSON, P.T.; YANG, M.; NIELSEN, H.M.; RANTANEN, J; FOGED, C. Stabilization of liposomes during drying. Expert Opinion Drug Delivery, v. 8 (3), p. 375-388, 2011.

MULLER, R.H.; RADTKE, M.; WISSING, S. Nanostructured lipid matrices for improved microencapsulation of drugs. International J. Pharmaceutics, v. 242, 121-128, 2002.

PARDEIKE, J.; WEBER, S.; HABER, T.; WAGNER, J.; ZARFL, H.P.; PLANK, H.; ZIMMER, A. Development of an itraconazole-loaded nanostructured lipid carrier formulation for pulmonary application. Int. J. Pharm., 419 (1-2), p. 329-338, 2011.

SARAF, A.S. Applications of novel drug delivery system for herbal formulations. Fitoterapia, v. 81, p. 680-689, 2010.

SINGH, S.; DOBHAL, A. K.; JAIN, A.; PANDIT, J. K.; CHAKRABORTY, S.; Chem. Pharm. Bull. (Tokyo), 58, 650, 2010.
SINGHAL, G.B.; PATEL, R.P.; PRAJAPATI, B.G.; PATEL, N.A. Solid lipid nanoparticles and nanostructured lipid carriers: as novel solid lipid based drug carrier. Int Res J Pharm., v. 2(2), p. 40-52, 2011.

SOUZA, C.R.F. Estudo comparativo da produção de extrato seco de Bauhinia forficata pelos processos spray dryer e leito de jorro. FCFRP/USP, 2003, 179p.

SOUZA, C.R.F.; OLIVEIRA, W.P. Microencapsulation of ketoprofen in blends of acrylic resins by spray drying. Drying Technology, v. 30 (3), p. 263-275, 2012.

WANG, T.; WANG, N.; ZHANG, Y.; SHEN, W.; GAO, X.; LI, T. Solvent injectionlyophilization of tert-butyl alcohol/water cosolvent systems for the preparation of drugloaded solid lipid nanoparticles. Colloids Surf. B, v. 79 (1), p. 254-261, 2010.

\section{AGRADECIMENTOS}

Os autores agradecem a FAPESP pela concessão de bolsa de estudo ao primeiro (Proc.\# 2014/01105-3) e último autores (Proc. \# 12/03427-2) e auxílio financeiro a pesquisa Projeto JP (Proc. \# 11/10333-1). 